\title{
Radiative Generation of Neutrino Masses and its Experimental Signals
}

\author{
K.S. Babu*t \\ Department of Physics, Oklahoma State University, Stillwater, OK 74078, USA \\ E-mail: babu@okstate.edu
}

\begin{abstract}
Tiny neutrino masses can arise naturally via loop diagrams. After a brief review of the radiative mass generation mechanism, I present a new model wherein $\mathrm{TeV}$ scale leptoquark scalars induce neutrino masses via two-loop diagrams. This model predicts the neutrino oscillation parameter $\sin ^{2} \theta_{13}$ to be close to the current experimental limit. The leptoquarks are accessible to experiments at the LHC since their masses must lie below $1.5 \mathrm{TeV}$, and their decay branching ratios probe neutrino oscillation parameters. Rare lepton flavor violating processes mediated by leptoquarks have an interesting pattern: $\mu \rightarrow e \gamma$ may be suppressed, while $\mu \rightarrow 3 e$ and $\mu-e$ conversion in nuclei are within reach of the next generation experiments. Muon $g-2$ receives new positive contributions, which can resolve the discrepancy between theory and experiment. New CP violating contributions to $B_{S}-\bar{B}_{s}$ mixing via leptoquark box diagrams are in a range that can explain the recently reported dimuon anomaly by the $\mathrm{D} \varnothing$ collaboration.
\end{abstract}

35th International Conference of High Energy Physics - ICHEP2010,

July 22-28, 2010

Paris France

\footnotetext{
* Speaker.

${ }^{\dagger}$ Work is supported in part by the US Department of Energy Grants DE-FG02-04ER41306 and DE-FG02-ER46140; OSU-HEP-11-2
} 
The standard paradigm for explaining tiny neutrino masses is the seesaw mechanism, which generates an effective dimension-5 operator $\mathscr{O}_{1}=(L L H H) / M$, suppressed by the mass scale $M$ of the heavy right-handed neutrino. ( $L$ here denotes lepton doublets, while $H$ is the Higgs doublet.) Oscillation data suggests that in this scenario $M \sim 10^{14} \mathrm{GeV}$, which is well beyond the reach of foreseeable experiments for direct scrutiny. An interesting alternative to the high scale seesaw mechanism is radiative mass generation. The smallness of neutrino masses can be understood as originating from loop and chirality suppression factors. The scale of new physics can naturally be around a $\mathrm{TeV}$ in this scenario. The simplest among this class of models is the Zee model [1] where neutrino masses are induced as one-loop radiative corrections arising from the exchange of charged scalar bosons. The effective lepton number violating operator in this model is $\mathscr{O}_{2}=L L L e^{c} H / M$. To convert this operator to neutrino mass, a loop diagram is necessary, as shown in the first diagram of Fig. 1. Here $\Phi_{1}$ is a charged scalar singlet transforming as $(1,1,1)$ of $S U(3)_{c} \times S U(2)_{L} \times U(1)_{Y}$ gauge symmetry and coupling to lepton doublets as $f_{i j} L_{i} L_{j} \Phi_{1}^{+}$with $f^{T}=-f . \Phi_{2}(1,2,-1 / 2)$ is a second Higgs doublet that generates charged lepton masses. The cubic scalar coupling $\Phi_{1} \Phi_{2} H$ in the scalar potential, along with the term $\left(\Phi_{2} H\right)^{2}$ ensure that lepton number is explicitly broken. The neutrino mass in this model is given by $m_{v} \sim\left(f m_{\ell}^{2}+m_{\ell}^{2} f^{T}\right) /\left(16 \pi^{2} M_{\Phi}\right)$, which for $M_{\Phi}=1 \mathrm{TeV}$ and $f=10^{-3}$ yields $m_{v} \sim 0.05 \mathrm{eV}$, of the right order to explain atmospheric neutrino oscillations. The simplest version of the Zee model is however excluded by neutrino oscillation data, since it predicts all the diagonal entries of the neutrino mass matrix to be zero, which is inconsistent.

In a second class of models, neutrino masses arise as two-loop radiative corrections [2] via the exchange of a singly charged scalar $\Phi_{1}(1,1,1)$ and a doubly charged scalar $\Phi_{2}(1,1,-2)$, as shown in the second diagram of Fig. 1. The Yukawa couplings $f_{i j} L_{i} L_{j} \Phi_{1}^{+}+g_{i j} e_{i}^{c} e_{j}^{c} \Phi_{2}^{--}$, with $f^{T}=-f, g^{T}=g$, along with the cubic scalar coupling $\Phi_{1}^{+} \Phi_{1}^{+} \Phi_{2}^{--}$ensure lepton number violation. The effective operator of this model is $\mathscr{O}_{9}=L L L e^{c} L e^{c} / M^{2}$, which requires two-loop dressing to convert to neutrino mass. Since $m_{v} \sim\left(f m_{\ell} g m_{\ell} f^{T}\right) /\left[\left(16 \pi^{2}\right)^{2} M_{\Phi}\right]$ in this model, for $f \sim g \sim 0.1$, and $M_{\Phi} \sim 1 \mathrm{TeV}, m_{v} \sim 0.05 \mathrm{eV}$ is generated. This model is consistent with neutrino oscillation data, and predicts the lightest neutrino to be nearly massless. Phenomenology of this model has been studied in Ref. [3]. The cross section for the production of a $1 \mathrm{TeV} \Phi_{2}^{--}$at the $\operatorname{LHC}(\sqrt{s}=14$ $\mathrm{TeV}$ ) is about $20 \mathrm{fb}$, which should be observable with its decay into same sign dileptons.
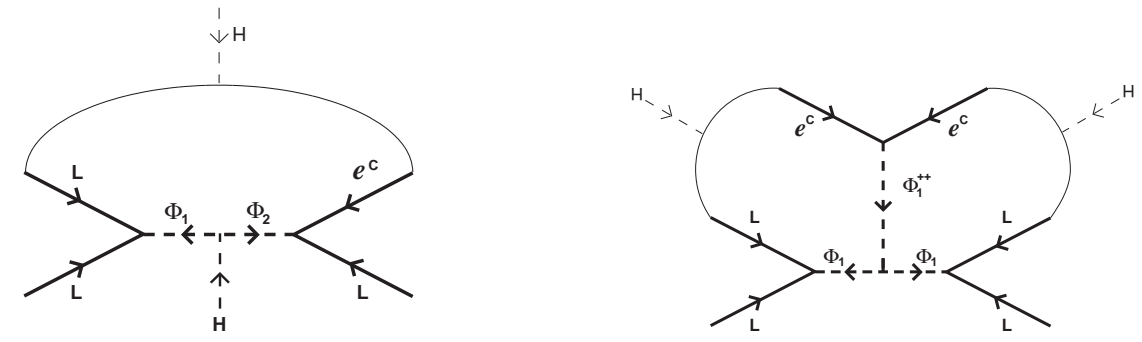

Figure 1: Loop diagrams generating small neutrino masses in the Zee model (left) and in the model of Ref. [2] (right).

A classification of low-dimensional effective $\Delta L=2$ lepton number violating operators that can lead to neutrino masses has been given in Ref. [4]. The list of operators includes $\mathscr{O}_{3}=$ $L^{i} L^{j} Q^{k} d^{c} H^{l} \varepsilon_{i k} \varepsilon_{j l}$, which appears in the context of $R$-parity violating supersymmetry. The operator $\mathscr{O}_{8}=L_{i} \overline{e^{c}} \overline{u^{c}} d^{c} H_{j} \varepsilon^{i j}$ is the subject for the remainder of this paper, which leads to an interesting neutrino mass model. $\mathscr{O}_{8}$ is most directly induced by the exchange of scalar leptoquarks (LQ). 
The order of magnitude of $m_{v}$ arising from $\mathscr{O}_{8}$ is $m_{v} \sim\left(m_{t} m_{b} m_{\tau} \mu v\right) /\left[\left(16 \pi^{2}\right)^{2} M_{\mathrm{LQ}}^{4}\right]$, where $\mu$ is the coefficient of a cubic scalar coupling, and $v=174 \mathrm{GeV}$ is the electroweak VEV. In order to generate $m_{v} \sim 0.05 \mathrm{eV}$, it is clear that $M_{\mathrm{LQ}}$ must be of order $\mathrm{TeV}$, which would be within reach of the LHC. The scalar sector consists of the leptoquark multiplets $\Omega(3,2,1 / 3) \equiv\left(\omega^{2 / 3}, \omega^{-1 / 3}\right)$ and $\chi^{-1 / 3}(3,1,-2 / 3)$. Assuming global baryon number conservation, the Lagrangian relevant for neutrino mass is

$$
\mathscr{L}_{v}=Y_{i j}\left(v_{i} d_{j}^{c} \omega^{-1 / 3}-\ell_{i} d_{j}^{c} \omega^{2 / 3}\right)+F_{i j} \ell_{i}^{c} u_{j}^{c} \chi^{-1 / 3}-\mu\left(\omega^{-2 / 3} H^{+}+\omega^{1 / 3} H^{0}\right) \chi^{-1 / 3}+\text { h.c. }
$$

The cubic scalar coupling will generate mixing between $\omega^{-1 / 3}$ and $\chi^{-1 / 3}$, we denote the mass eigenstates $X^{a}$, their masses $M_{1,2}$, and the mixing angle $\theta$. Neutrino masses are induced via Fig. 2 .

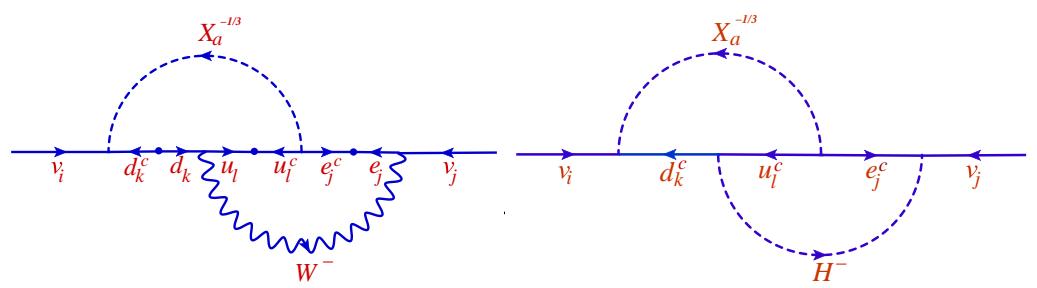

Figure 2: Two-loop diagrams contributing to neutrino mass generation via $\mathscr{O}_{8}$.

Combining constraints from flavor changing processes, we find the neutrino mass matrix to be

$$
M_{V} \simeq m_{0}\left(\begin{array}{ccc}
0 & \frac{1}{2} \frac{m_{\mu}}{\frac{m_{\tau}}{m_{\tau}} x y} & \frac{1}{2} y \\
\frac{1}{2} \frac{m_{\mu}}{m_{\tau}} x y & \frac{m_{\mu}}{m_{\tau}} x z & \frac{1}{2} z+\frac{1}{2} \frac{m_{\mu}}{m_{\tau}} x \\
\frac{1}{2} y & \frac{1}{2} z+\frac{1}{2} \frac{m_{\mu}}{m_{\tau}} x & 1+w
\end{array}\right) .
$$

Here $x=F_{23}^{*} / F_{33}^{*}, y=Y_{13} / Y_{33}, z=Y_{23} / Y_{33}, w=\left(F_{32}^{*} / F_{33}^{*}\right)\left(Y_{32} / Y_{33}\right)\left(m_{c} / m_{t}\right)\left(m_{s} / m_{b}\right)\left(I_{j k 2} / I_{j k 3}\right)$ and $m_{0}=\left(6 g^{2} \sin 2 \theta F_{33}^{*} Y_{33} I_{j k 3}\right)\left(m_{t} m_{b} m_{\tau}\right) /\left[\left(16 \pi^{2}\right)^{2} M_{1}^{2}\right] . I_{j k 3}$ denotes the two loop integral function shown in Fig. 3, with the internal up-type quark being the top. Since the $(1,1)$ entry is zero, and
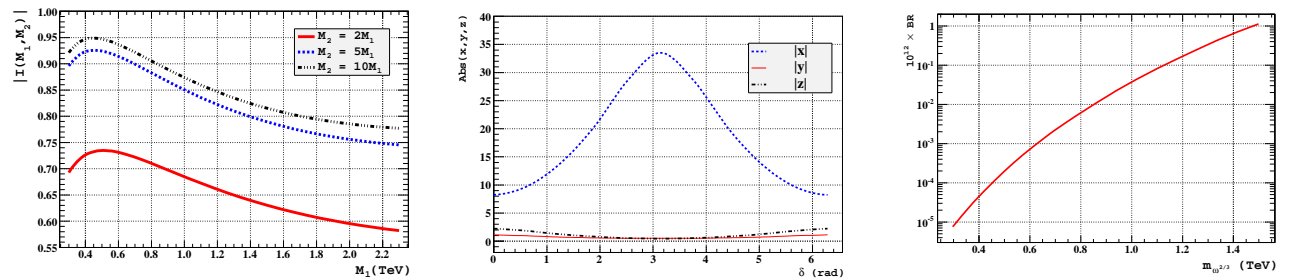

Figure 3: The integral $I_{j k 3}$ versus $M_{1}$ (left); $|x|,|y|,|z|$ versus $\delta$ (middle); $\mu \rightarrow 3 e$ branching ratio versus $m_{\omega^{2 / 3}}$ (right).

$w$ is highly suppressed, the determinant of $M_{v}$ is nearly zero. This leads to the predictions $m_{1} \simeq 0$, and $\tan ^{2} \theta_{13} \simeq m_{2} / m_{3} \sin ^{2} \theta_{12}$ in the standard parametrization of neutrino mixing. This leads to $\sin ^{2} \theta_{13}=(0.044-0.051)$, which is near the current limit. A consistent fit to global oscillation parameters is obtained. The parameters $x, y, z$ for such a fit are plotted as functions of the unknown $\mathrm{CP}$ violating phase $\delta$ in Fig. 3 (middle panel). We see that $|x| \gg 1$ and $|y|,|z| \sim 1$. These values fix the branching ratios of the leptoquarks: $\Gamma\left(\omega^{2 / 3} \rightarrow e^{+} b\right): \Gamma\left(\omega^{2 / 3} \rightarrow \mu^{+} b\right): \Gamma\left(\omega^{2 / 3} \rightarrow \tau^{+} b\right)=$ $|y|^{2}:|z|^{2}: 1$, and $\Gamma\left(X_{a}^{-1 / 3} \rightarrow \mu^{-} t\right): \Gamma\left(X_{a}^{-1 / 3} \rightarrow \tau^{-} t\right)=|x|^{2}: 1$. Measuring these decays will 
thus probe $\mathrm{CP}$ violation in neutrino oscillations. From the experimental limit on $\mu \rightarrow 3 e$ and $\mu-e$ conversion in nuclei, we derive the upper limit on $\left|Y_{13}^{*} Y_{23}\right|$ as a function of $\omega^{2 / 3}$ mass. Since neutrino oscillation data requires $Y_{i 3}$ to be of the same order for $i=1-3$, one can also determine an upper limit on $Y_{33}$. Combining these, we obtain an upper limit of $1.5 \mathrm{TeV}$ on $M_{1}$, as shown in Fig. 3 as a function of $\mu \rightarrow 3 e$ branching ratio (right panel).

Since the leptoquarks of the model are at the TeV scale, they can mediate lepton flavor violation through the $Y_{i j}$ and $F_{i j}$ couplings. Experimental limits on these couplings have been satisfied in our neutrino fit. The processes we consider are $\mu^{-} \rightarrow e^{-} \gamma, \mu^{-} \rightarrow e^{+} e^{-} e^{-}, \mu-e$ conversion in nuclei, $\tau^{-} \rightarrow e^{-} \eta, \tau^{-} \rightarrow \mu^{-} \eta, B_{s, d}-\bar{B}_{s, d}$ mixing, $K-\bar{K}$ mixing, $D-\bar{D}$ mixing, $D_{s}^{ \pm} \rightarrow \ell^{ \pm} v$ decay, muon $g-2, \pi^{+} \rightarrow \mu^{+} \overline{v_{e}}$ decay, and neutrinoless double beta decay. Interestingly, we find that $\mu \rightarrow e \gamma$ mediated by the $\omega$ LQ is suppressed by a GIM-like mechanism. For the $\chi$ LQ mass of $1 \mathrm{TeV}$, we obtain from $\mu \rightarrow e \gamma,\left|\sum_{i} F_{1 i}^{*} F_{2 i}\right| \leq 6.7 \times 10^{-3}$, but no constraint for the $\omega \mathrm{LQ}$. If all the LQ masses are $1 \mathrm{TeV}$, we obtain from $\mu \rightarrow 3 e$ the limits $\left|Y_{13} Y_{23}\right|<7.6 \times 10^{-3},\left|F_{13} F_{23}\right|<1.8 \times 10^{-3}$, and from $\mu-e$ conversion in nuclei slightly better limits $\left|Y_{13} Y_{23}\right|<4.6 \times 10^{-3},\left|F_{13} F_{23}\right|<1.9 \times 10^{-4}$. The decays $\tau^{-} \rightarrow e^{-} \eta$ and $\tau^{-} \rightarrow \mu^{-} \eta$ provide the limits $\left|Y_{12} Y_{32}\right|<1.2 \times 10^{-2},\left|Y_{22} Y_{32}\right|<1.0 \times 10^{-2}$, now for the $\omega$ LQ mass of 300 $\mathrm{GeV} . g-2$ of the muon receives new positive contributions from the $\chi \mathrm{LQ}$, which can be as large as $\delta(g-2) \approx 12 \times 10^{-10}$ for $\chi$ mass of $300 \mathrm{GeV}$. This will be nicely consistent with the indication that $\delta(g-2)=(24.6 \pm 8.0) \times 10^{-10}$.

Recently the DØ Collaboration has reported a 3.2 sigma excess in the like-sign dimuon asymmetry compared to theory. A likely explanation is that there is a new source of $\mathrm{CP}$ violation in $B_{s}-\bar{B}_{s}$ mixing, which can arise from leptoquark box diagrams. If the $B_{s}-\bar{B}_{s}$ mass difference is written as $\Delta M_{s}=\Delta M_{s}^{\mathrm{SM}}\left|1+h_{s} e^{2 i \sigma_{s}}\right|$, then a good fit to the data is obtained for [6] $\left\{h_{s} \sim 0.5, \sigma_{s} \sim 120^{\circ}\right\}$ or $\left\{h_{s} \sim 1.8, \sigma_{s} \sim 100^{\circ}\right\} . h_{s} \sim 0.5$ is realized in our model for $\left|Y_{32}\right| \sim 1$ and $m_{\omega}=390 \mathrm{GeV}$ or $\left|Y_{32}\right| \sim 0.77$ and $m_{\omega}=300 \mathrm{GeV}$. The phase $\sigma_{s}$ is unconstrained. This scenario will predict the branching ratio for $B_{s} \rightarrow \tau^{+} \tau^{-}$at the $0.25 \%$ level, compared to the standard model value of $10^{-6}$. Finally, neutrinoless double beta decay proportional to neutrino mass is suppressed in this model. However, it can proceed via the vector-scalar exchange process [7]. The diagram involves exchange of one leptoquark and one $W$ boson. We obtain the constraint $\left|Y_{11}^{*} F_{11}\right|<1.7 \times 10^{-6}\left(\frac{M_{1}}{1 \mathrm{TeV}}\right)^{2}\left(\frac{0.5 \mathrm{TeV}}{\mu}\right)$ from this process, indicating that neutrinoless double beta decay may be observable, in spite of the mass hierarchy being normal.

\section{References}

[1] A. Zee, Phys. Lett. B93, 389 (1980).

[2] A. Zee, Nucl. Phys. B 264, 99 (1986); K. S. Babu, Phys. Lett. B203, 132 (1988).

[3] K. S. Babu and C. Macesanu, Phys. Rev. D 67, 073010 (2003); M. Nebot, J. F. Oliver, D. Palao, and A. Santamaria, Phys. Rev. D 77, 093013 (2008); D. A. Sierra and M. Hirsch, JHEP 0612, 052 (2006).

[4] K. S. Babu and C. N. Leung, Nucl. Phys. B619, 667 (2001).

[5] K. S. Babu and J. Julio, Nucl. Phys. B 841, 130 (2010).

[6] Z. Ligeti, M. Papucci, G. Perez and J. Zupan, Phys. Rev. Lett. 105, 131601 (2010).

[7] K. S. Babu and R. N. Mohapatra, Phys. Rev. Lett. 75, 2276 (1995). 